CERN-TH/96-201

SNUTP 96-79

hep-th/9607243

\title{
Gravitational Dressing of Massive Soliton Theories
}

\author{
Ioannis Bakas 周间 \\ Theory Division, CERN, 1211 Geneva 23, Switzerland \\ Q-Han Park f \\ Department of Physics, and Research Institute of Basic Sciences \\ Kyunghee University, Seoul, 130-701, Korea
}

\begin{abstract}
The massive soliton theories describe integrable perturbations of WZW cosets as generalized multi-component sine-Gordon models. We study their coupling to 2-dim gravity in the conformal gauge and show that the resulting models can be interpreted as conformal non-Abelian Toda theories when a certain algebraic condition is satisfied. These models, however, do not provide quantum mechanically consistent string backgrounds in the case the underlying WZW constraints are first solved classically.
\end{abstract}

July 1996

* Permanent address: Department of Physics, University of Patras, 26110 Patras, Greece

†e-mail address: BAKAS@NXTH04.CERN.CH

$\ddagger$ e-mail address: QPARK@NMS.KYUNGHEE.AC.KR 
It is well known that any Lagrangian can be made scale invariant by appropriate coupling to a bosonic field $\phi$ that transforms non-linearly $\phi(x) \rightarrow \phi\left(e^{\alpha} x\right)+\alpha / \gamma$, where $\gamma$ is a constant and $\alpha$ is the scale parameter. Let $L=L_{s}-V$ be the decomposition of the original Lagrangian into a scale invariant part $L_{s}$ and a scale breaking potential $V$. Then,

$$
L^{\prime}=L_{s}-e^{2 \gamma \phi} V+\frac{1}{2 \gamma^{2}} \partial_{\mu} e^{\gamma \phi} \partial^{\mu} e^{\gamma \phi}
$$

is manifestly scale invariant and also conformal. This prescription was used in the past to write down 4-dim phenomenological Lagrangians for which mesons and nucleons have non-zero mass in lowest order perturbation theory and only $\phi$ is massless serving in this case as the Goldstone boson of scale invariance (see for instance [1] and references therein).

The above procedure has a natural interpretation in two dimensions by providing the coupling of a relativistic theory to gravity in the conformal gauge. For instance, we may think of $L$ as an integrable perturbation of a conformal field theory, typically given by a gauged WZW Lagrangian $L_{s}$ plus a potential term $V$ that breaks conformal invariance while preserving integrability à la Zamolodchikov [2]. The coupling to 2-dim gravity amounts to choosing a metric $g=e^{2 \gamma \phi} \widehat{g}$ with the corresponding action becoming

$$
S^{\prime}=S_{s}-\int d^{2} z \sqrt{\widehat{g}} e^{2 \gamma \phi} V+S_{L},
$$

where the contribution of the Liouville field $\phi$ is

$$
S_{L}=\frac{1}{\pi} \int d^{2} z \sqrt{\widehat{g}}\left(\frac{1}{2} \widehat{g}^{a b} \partial_{a} \phi \partial_{b} \phi+Q \phi R(\widehat{g})+\mu e^{2 \gamma \phi}\right)
$$

The contribution of the dilaton term vanishes by specializing to flat $\widehat{g}_{a b}$, while the conformal factor transforms as usual under $z \rightarrow f(z)$,

$$
\phi(z) \rightarrow \phi(f(z))+\frac{1}{2 \gamma} \log |\partial f|^{2},
$$

in terms of light-cone variables. The cosmological term $\mu$ arises due to the freedom to shift $V$ by a constant that changes the zero point energy without affecting the classical equations of motion of the system before coupling it to gravity. This is the general framework of the gravitational dressing; see the original papers [3] for further details.

There are already some results in the literature on the gravitational sine-Gordon model, which arises by perturbing the free field action by a cosine potential and then coupling it to the Liouville theory as above [4, 5]. Here we consider the more general situation of a 2-dim relativistic system described by a conformal coset model $G / H$, plus an integrable perturbation, and determine the circumstances under which the coupling to gravity preserves the integrability at least classically. The most typical example in our scheme, besides the simplest sine-Gordon model, is the so called complex sine-Gordon (here we actually consider its analytic continuation with hyperbolic elements) with Lagrangian

$$
L=\partial r \bar{\partial} r-\tanh ^{2} r \partial \theta \bar{\partial} \theta+\lambda \cosh 2 r
$$


with coupling constant $\lambda$. This model was recently interpreted as an integrable perturbation of the WZW coset $S L(2) / U(1)$ by the first thermal (parafermionic) operator $\epsilon_{1}$ that turns on the potential term $\cosh 2 r[6,7]$; similar results hold for the compact coset $S U(2) / U(1)$. Upon gravitational dressing, according to the general framework, the resulting conformal theory for appropriately chosen constants $\gamma$ and $\lambda$ assumes the form

$$
L^{\prime}=\partial r \bar{\partial} r-\tanh ^{2} r \partial \theta \bar{\partial} \theta+\partial \phi \bar{\partial} \phi+e^{2 \phi} \cosh 2 r
$$

which is easily recognized as the non-Abelian $B_{2}$ Toda model [8, 9]. Non-Abelian Toda theories will be the center theme of our study later. They were also investigated before as exactly solvable conformal systems of the matter field $\phi$ in the presence of blackhole backgrounds (the simplest example being the semi-classical geometry of the coset $S L(2) / U(1)$ ) [9], but their interpretation in terms of the gravitational dressing of integrable perturbations of coset models appears to be new in the present context.

Before proceeding further we note for completeness that apart from the ordinary sineGordon model various aspects of some other gravitationally dressed relativistic integrable systems have also been considered elsewhere: for the Gross-Neveu model coupling to gravity in the chiral (Polyakov) gauge [10], and for the principal chiral model in the conformal gauge [11]. The theories we consider below form a different class that is rather wide. A primary aim is to accumulate further results in the developing area of gravitationally dressed systems by encompassing integrable models like (5), (6), and multi-component generalizations thereof, thus establishing new connections among them in terms of 2-dim gravity.

The general framework of the present work is given by the class of massive integrable soliton theories (MIST), as was formulated in [12], and their algebraic connection with non-Abelian Toda theories. Recall first the essential technical ingredients that define the relevant class of integrable models. We consider the $s l(2)$ embedding of a finite algebra g specified by the generators $\left\{J_{ \pm}, J_{0}\right\}$ with

$$
\left[J_{0}, J_{ \pm}\right]= \pm J_{ \pm}, \quad\left[J_{+}, J_{-}\right]=2 J_{0}
$$

so that the Cartan element $J_{0}$ induces a gradation of $\mathbf{g}$ as follows:

$$
\mathbf{g}=\mathbf{g}_{-1} \oplus \mathbf{g}_{0} \oplus \mathbf{g}_{1} ; \quad\left[J_{0}, \mathbf{g}_{k}\right]=k \mathbf{g}_{k}, \quad k=0, \pm 1
$$

In this case we speak of an $N=1$ grading of $\mathbf{g}$ and $\mathbf{g}_{0}$, which is the zero graded part of $\mathbf{g}$, forms a subalgebra. More general gradations correspond to the decomposition $\mathbf{g}=\oplus_{k=-N}^{k=N} \mathbf{g}_{k}$, but these will not enter at all into the present work. Let $G_{0}$ denote the Lie group associated with $\mathbf{g}_{0}$ and write down the action

$$
S=S_{W Z W}(g, A, \bar{A})+\frac{m^{2}}{2 \pi} \int \operatorname{Tr}\left(g^{-1} T g \bar{T}\right)
$$

where $g$ is an element of $G_{0}$ and $T, \bar{T}$ are constant elements of the Lie algebra $\mathbf{g}$ (though they remain arbitrary at the moment). The term $S_{W Z W}$ is the usual gauged WZW 
action $(1 / 4 \pi) \int \operatorname{Tr}\left(g^{-1} \partial g g^{-1} \bar{\partial} g\right)+W Z$-term $+(A, \bar{A})$-terms, where $\operatorname{Tr}\left(T^{a} T^{b}\right)=2 \delta^{a b}$. The gauge connections $A, \bar{A}$ take values in the subgroup $H$ of $G_{0}$ whose algebra is defined by

$$
\mathbf{h}=\left\{v \in \mathbf{g}_{0} ; \quad[v, T]=0=[v, \bar{T}]\right\}
$$

This on the other hand specifies the flat directions of the potential in (9), since the potential term is invariant under the adjoint action of $H$ associated with the Lie algebra h.

For the WZW model $G_{0} / H$, where $H$ is assumed to be diagonal subgroup of $G_{0}, A$ and $\bar{A}$ act as Lagrange multipliers resulting in the constraint equations

$$
\begin{aligned}
\delta_{A} S & =\frac{1}{2 \pi} \int \operatorname{Tr}\left(-\bar{\partial} g g^{-1}+g \bar{A} g^{-1}-\bar{A}\right) \delta A=0 \\
\delta_{\bar{A}} S & =\frac{1}{2 \pi} \int \operatorname{Tr}\left(g^{-1} \partial g+g^{-1} A g-A\right) \delta \bar{A}=0
\end{aligned}
$$

that remove the flat directions of the potential. The remaining equations of motion take the form

$$
\left[\bar{\partial}+\bar{A}, g^{-1} D g\right]=m^{2}\left[T, g^{-1} \bar{T} g\right]
$$

or in zero curvature form they become

$$
\left[\partial+g^{-1} D g+l T, \bar{\partial}+\bar{A}+\frac{m^{2}}{l} g^{-1} \bar{T} g\right]=0,
$$

where $g^{-1} D g=g^{-1} \partial g+g^{-1} A g$ and $l$ is a spectral parameter. Projecting (13) in $\mathbf{h}$ and using the constraint equations it follows that the gauge field is flat, $[\partial+A, \bar{\partial}+\bar{A}]=0$, which in turn allows for the particularly simple gauge fixing

$$
A=0=\bar{A}
$$

We then arrive at the equations

$$
\bar{\partial}\left(g^{-1} \partial g\right)=m^{2}\left[T, g^{-1} \bar{T} g\right] ; \quad\left(g^{-1} \partial g\right)_{\mathbf{h}}=0=\left(\bar{\partial} g g^{-1}\right)_{\mathbf{h}}
$$

taking into account the projection in $\mathbf{h}$ as it is explicitly indicated in (16).

Within the above general framework we may specialize the class of integrable field theories (9) according to specific choices for $T$ and $\bar{T}$. Here we will consider two cases:

(a) Massive models, choosing $T=\bar{T}=J_{+}+J_{-}$for which the potential explicitly breaks conformal invariance acting as a mass term. We obtain in this case soliton equations that provide a non-Abelian extension of the sine-Gordon model to soliton theories with internal degrees of freedom $[12,13]$. The complete list of the massive integrable soliton theories associated to $N=1$ grading of all classical Lie algebras $\mathbf{g}$ (MIST) has been obtained in [12]. These models are invariant under parity.

(b) Conformal models, choosing $T=J_{+}$and $\bar{T}=J_{-}$. In this case the potential term possesses the right scaling properties for defining conformally invariant models known as non-Abelian Toda theories [8, 9] generalizing the Liouville theory. 
In both cases above the non-Abelian nature of the models originates from the nonAbelian algebra $\mathbf{g}_{0}$ in the $N=1$ grading (8) of $\mathbf{g}$. The affine (non-conformal) Toda theories correspond to the choice $T=J_{+}+Y_{+}$and $\bar{T}=J_{-}+Y_{-}$, where $Y_{ \pm}$are elements of the maximally graded parts $\mathbf{g}_{\mp N}$ for a general gradation. Hence, the models (a) are specified by the choice $Y_{ \pm}=J_{\mp}$.

A crucial property inherited from this algebraic model building is the factorization of $G_{0}$ into a direct product of an abelian $U(1)$ factor generated by $J_{0}$ of $s l(2)$ and the remaining (non-abelian) group denoted by $G_{0}^{\prime}$. This decomposition is the starting point for further refining the class of MIST under consideration. In fact we will consider next those models for which the field $\chi$ associated with the abelian $U(1)$ factor can be decoupled consistently. We will derive the algebraic condition that is necessary for reducing the MIST models by setting $\chi=0$ while maintaining consistency with the classical equations of motion. The complex sine-Gordon model can be obtained precisely in this fashion, but now within the present framework we have a whole class of possible massive multi-component generalizations as well. Upon gravitational dressing, as we will see later, this particular class of models admits a systematic description as conformal models of type (b), i.e. non-Abelian Toda theories; the simplest example of such a construction/correspondence is the transition from (5) to (6), which was considered before as motivation. This also explains why we have presented here a unified description of the models (a) and (b).

Let $g=g^{\prime} \exp \left(i \chi J_{0}\right)$ be the decomposition of a group element in $G_{0}$ into $G_{0}^{\prime}$ and the commuting $U(1)$ factor generated by $J_{0}$. Then, the equations of motion (16) of any MIST with $T=\bar{T}=J_{+}+J_{-}$decompose as follows:

$$
\begin{aligned}
\bar{\partial}\left(g^{-1} \partial g\right) & =\bar{\partial}\left(g^{\prime-1} \partial g^{\prime}\right)+i \bar{\partial} \partial \chi J_{0}=m^{2}\left[J_{+}+J_{-}, g^{-1}\left(J_{+}+J_{-}\right) g\right] \\
& =m^{2} e^{i \chi}\left[J_{+}, g^{\prime-1} J_{-} g^{\prime}\right]+m^{2} e^{-i \chi}\left[J_{-}, g^{\prime-1} J_{+} g^{\prime}\right]
\end{aligned}
$$

where we used the fact that $g^{\prime}$ commutes with $J_{0}$. Then, the $U(1)$ part of this equation yields

$$
\begin{aligned}
i \bar{\partial} \partial \chi & =\frac{m^{2}}{\operatorname{Tr} J_{0}^{2}} \operatorname{Tr} J_{0}\left(e^{i \chi}\left[J_{+}, g^{\prime-1} J_{-} g^{\prime}\right]+e^{-i \chi}\left[J_{-}, g^{\prime-1} J_{+} g^{\prime}\right]\right) \\
& =\frac{m^{2}}{\operatorname{Tr} J_{0}^{2}} \operatorname{Tr}\left(e^{i \chi} J_{+} g^{\prime-1} J_{-} g^{\prime}-e^{-i \chi} J_{-} g^{\prime-1} J_{+} g^{\prime}\right) .
\end{aligned}
$$

Thus, the $U(1)$ factor can be consistently decoupled by setting $\chi=0$ without contradicting the classical equations of motion provided that the particular MIST model satisfies the condition

$$
\operatorname{Tr}\left(J_{+} g^{-1} J_{-} g^{\prime}\right)=\operatorname{Tr}\left(J_{-} g^{-1} J_{+} g^{\prime}\right) .
$$

The models that result in this fashion are integrable having as equations of motion

$$
\bar{\partial}\left(g^{\prime-1} \partial g^{\prime}\right)=m^{2}\left(\left[J_{+}, g^{\prime-1} J_{-} g^{\prime}\right]+\left[J_{-}, g^{\prime-1} J_{+} g^{\prime}\right]\right) .
$$


Starting now from any consistently reduced MIST model with $\chi=0$, the coupling to 2-dim gravity in the conformal gauge $g_{a b}=e^{2 \phi} \delta_{a b}$ amounts to the conformally invariant theory

$$
S^{\prime}=S_{W Z W}\left(g^{\prime}, A, \bar{A}\right)+\frac{2}{\pi} \int d^{2} z \partial \phi \bar{\partial} \phi+\frac{m^{2}}{\pi} \int d^{2} z e^{2 \phi} \operatorname{Tr}\left(J_{+} g^{\prime-1} J_{-} g^{\prime}\right)
$$

where we used the special condition (19) for the original potential term to write down $\operatorname{Tr}\left(\left(J_{+}+J_{-}\right) g^{\prime-1}\left(J_{+}+J_{-}\right) g^{\prime}\right)=2 \operatorname{Tr}\left(J_{+} g^{\prime-1} J_{-} g^{\prime}\right)$. Notice that $\operatorname{Tr}\left(J_{+} g^{\prime-1} J_{+} g^{\prime}\right)=0$ using (7) and the commutativity of $J_{0}$ with $g^{\prime}$; indeed $\operatorname{Tr}\left(J_{+} g^{\prime-1} J_{+} g^{\prime}\right)=\operatorname{Tr}\left(\left[J_{0}, J_{+}\right] g^{\prime-1} J_{+} g^{\prime}\right)=$ $\operatorname{Tr}\left(J_{+} g^{\prime-1}\left[J_{+}, J_{0}\right] g^{\prime}\right)=-\operatorname{Tr}\left(J_{+} g^{\prime-1} J_{+} g^{\prime}\right)$, and so this term vanishes. We may prove similarly that $\operatorname{Tr}\left(J_{-} g^{\prime-1} J_{-} g^{\prime}\right)=0$. At this point we introduce group elements

$$
\tilde{g}=g^{\prime} e^{2 \phi J_{0}}
$$

and note that

$$
e^{2 \phi} \operatorname{Tr}\left(J_{+} g^{\prime-1} J_{-} g^{\prime}\right)=\operatorname{Tr}\left(e^{2 \phi J_{0}} J_{+} e^{-2 \phi J_{0}} g^{\prime-1} J_{-} g^{\prime}\right)=\operatorname{Tr}\left(J_{+} \tilde{g}^{-1} J_{-} \tilde{g}\right)
$$

As a result the conformal theory (21) becomes

$$
S^{\prime}=S_{W Z W}(\tilde{g}, A, \bar{A})+\frac{m^{2}}{\pi} \int d^{2} z \operatorname{Tr}\left(J_{+} \tilde{g}^{-1} J_{-} \tilde{g}\right)
$$

which is immediately recognized as a non-Abelian Toda theory of type (b) with $T=J_{+}$, $\bar{T}=J_{-}$. This result summarizes the effect of the gravitational dressing on the class of integrable models satisfying the algebraic condition (19). Alternatively, it provides a new geometrical interpretation to a wide class of conformal non-Abelian Toda models. It is also interesting to recall that the massive soliton theories before dressing them with gravity describe integrable perturbations of WZW models away from criticality. We will present some examples later.

Note that despite of appearances the field $\chi$ (before decoupling it from a massive integrable soliton theory) and the Liouville field $\phi$ (after dressing the resulting models with 2-dim gravity) play a different role: we may effectively interpret $\chi$ as providing a sine-Gordon type coupling, hence leading to the massive integrable soliton theories with broken conformal invariance, as in (17), while $\phi$ is a Liouville coupling restoring the conformal invariance classically. Apart from this, the field $\phi$ satisfies a field equation following (21) that renders the choice $\phi=0$ inconsistent; a consistent choice is instead $\phi \rightarrow-\infty$. However, as far as the WZW part of the action (24) is concerned, it is obvious that $S_{W Z W}(g, A, \bar{A})$ is essentially the same as $S_{W Z W}(\tilde{g}, A, \bar{A})$, modulo a formal analytic continuation $\chi \rightarrow-2 i \phi$ that changes the commuting $U(1)$ factor of $G_{0}$ into the noncompact form $R$. The potential terms on the other hand are not equivalent; they agree only when the group elements take values in $G_{0}^{\prime}$, i.e. $\operatorname{Tr}\left(J_{+} g^{-1} J_{-} g^{\prime}\right)$. Of course, $T$ and $\bar{T}$ are identified differently in each case. This comparison is useful to understand intuitively the essence of our results without willing to threaten them with trivialization.

The resulting non-Abelian Toda theories provide a class of curved backgrounds for string propagation in the presence of a tachyon field, which is identified with the Toda 
potential. A drawback of these models, in their formulation obtained by solving classically the WZW constraints, is that they are not conformal quantum mechanically and therefore fail to be solutions of the corresponding $\beta$-function equations; we will elaborate more on this issue towards the end of the paper. Thus, our interest in the classically reduced form of the models is only from the point of view of integrable 2-dim field theories and not for applications in string theory.

Next, we examine for which algebras $\mathbf{g}$ with $N=1$ grading the decoupling condition (19) is satisfied, and also present a few explicit examples illustrating the general situation.

(i) A-series: For the $A$-series the rank of the algebra $\mathbf{g}$ has to be odd, and so we consider $A_{2 n-1}$ for which there is an $N=1$ embedding of $s l(2)$ specified in the defining representation by

$$
J_{0}=\frac{1}{2}\left(\begin{array}{cc}
1_{n} & 0 \\
0 & -1_{n}
\end{array}\right), \quad J_{+}=\left(\begin{array}{cc}
0 & 1_{n} \\
0 & 0
\end{array}\right), \quad J_{-}=\left(\begin{array}{cc}
0 & 0 \\
1_{n} & 0
\end{array}\right) .
$$

This results in the zero-graded part of the algebra $\mathbf{g}_{0}=s u(n) \oplus s u(n) \oplus u(1)$ and $\mathbf{h}=s u(n)$ commuting with $T=\bar{T}=J_{+}+J_{-}$, which are represented respectively by

$$
\left(\begin{array}{ll}
a & 0 \\
0 & b
\end{array}\right), a^{\dagger}=a, b^{\dagger}=b, \quad \operatorname{Tr}(a+b)=0 ; \quad\left(\begin{array}{ll}
a & 0 \\
0 & a
\end{array}\right), a^{\dagger}=a, \quad \operatorname{Tr} a=0 .
$$

So the corresponding MIST models are integrable perturbations of the WZW coset

$$
\frac{S U(n) \times S U(n)}{S U(n)} \times U(1)
$$

and the potential term is

$$
\operatorname{Tr}\left(g^{-1} T g \bar{T}\right)=\operatorname{Tr}\left(g_{1}^{\prime} g_{2}^{\prime-1}+g_{2}^{\prime} g_{1}^{\prime-1}\right)
$$

by restricting to group elements $g^{\prime}=\left(g_{1}^{\prime}, g_{2}^{\prime}\right)$ in $G_{0}^{\prime}=S U(n) \times S U(n)$ when $\chi=0$. To proceed with the gravitational dressing of the resulting integrable models one has to check the validity of the decoupling condition (19) in this case. It can be easily verified that this condition is satisfied only for $n=1$ and $n=2$ since then $\operatorname{Tr}\left(g_{1}^{\prime} g_{2}^{\prime-1}\right)=\operatorname{Tr}\left(g_{2}^{\prime} g_{1}^{\prime-1}\right)=$ $\operatorname{Tr}\left(g_{1}^{\prime} g_{2}^{\prime-1}\right)^{-1}$. We will examine each case separately.

For $A_{1}(n=1)$ the massive integrable soliton theory with $\chi=0$ is precisely the sine-Gordon model

$$
\bar{\partial} \partial \theta=2 m^{2} \sin 2 \theta,
$$

where $g_{1}^{\prime}=e^{i \theta}, g_{2}^{\prime}=e^{-i \theta}$ is a good parametrization that solves the WZW constraints in (16). If we dress it gravitationally using the parametrization

$$
\tilde{g}=\left(\begin{array}{cc}
e^{i \theta} & 0 \\
0 & e^{-i \theta}
\end{array}\right) e^{2 \phi J_{0}}=\left(\begin{array}{cc}
e^{i \theta+\phi} & 0 \\
0 & e^{-i \theta+\phi}
\end{array}\right),
$$


the resulting conformal model is the complexified Liouville theory whose real and imaginary parts are respectively

$$
\bar{\partial} \partial \phi=2 m^{2} e^{2 \phi} \cos 2 \theta ; \quad \bar{\partial} \partial \theta=2 m^{2} e^{2 \phi} \sin 2 \theta .
$$

For $A_{3}(n=2)$, setting $\chi=0$, we arrive at the so-called matrix sine-Gordon model (see [13] for details) for which the appropriate parametrization of $g_{1}^{\prime}$ and $g_{2}^{\prime}$ in $S U(2)$ can be found by solving the corresponding WZW constraints

$$
g_{1}^{\prime-1} \partial g_{1}^{\prime}+g_{2}^{\prime-1} \partial g_{2}^{\prime}=0, \quad \bar{\partial} g_{1}^{\prime} g_{1}^{\prime-1}+\bar{\partial} g_{2}^{\prime} g_{2}^{\prime-1}=0
$$

Then, its gravitational dressing proceeds as above to yield the coupled system of equations

$$
\bar{\partial}\left(g_{1}^{\prime-1} \partial g_{1}^{\prime}\right)=m^{2} e^{2 \phi}\left(g_{1}^{\prime-1} g_{2}^{\prime}-g_{2}^{\prime-1} g_{1}^{\prime}\right), \quad \bar{\partial} \partial \phi=m^{2} e^{2 \phi} \operatorname{Tr}\left(g_{1}^{\prime-1} g_{2}^{\prime}+g_{2}^{\prime-1} g_{1}^{\prime}\right) \text {. }
$$

(ii) $C$-series: For $C_{n}$ there is only one $N=1$ embedding of $s l(2)$ specified in the defining representation by $(25)$ as before. Then, the zero-graded subalgebra is $\mathbf{g}_{0}=$ $s u(n) \oplus u(1)$ and $\mathbf{h}=s o(n)$, which are represented respectively by

$$
\left(\begin{array}{cc}
a & 0 \\
0 & -a^{\star}
\end{array}\right), \quad a^{\dagger}=a ; \quad\left(\begin{array}{cc}
a & 0 \\
0 & a
\end{array}\right), \quad a^{\star}=-a, \quad a^{t}=-a .
$$

As a result, the corresponding MIST models are integrable perturbations of

$$
\frac{S U(n)}{S O(n)} \times U(1)
$$

and the potential term takes the form

$$
\operatorname{Tr}\left(g^{-1} \operatorname{Tg} \bar{T}\right)=\operatorname{Tr}\left(g^{\prime} g^{\prime t}+g^{\prime \star} g^{\prime-1}\right)
$$

where the group elements are restricted to $\left(g^{\prime}, g^{\prime \star}\right)$ in the $2 n$-dim representation of $G_{0}^{\prime}=S U(n)$ (with $g^{\prime} \in S U(n)$ ) by setting $\chi=0$. It is a matter of straightforward calculation to verify that the special condition (19) is satisfied only for $n=1$ and $n=2$, as in the $A$-series above, since only for them we have $\operatorname{Tr}\left(g^{\prime} g^{\prime-1^{\star}}\right)=\operatorname{Tr}\left(g^{\prime \star} g^{\prime-1}\right)$.

The case $n=1$ is trivial reducing to the $A_{1}$ sine-Gordon model as before, while $C_{2}$ provides the only model in this series that is eligible to yield a non-Abelian Toda theory upon gravitational dressing. The resulting conformal theory coincides with the gravitationally dressed complex sine-Gordon, which can also be considered, as we will see next, as the non-Abelian Toda theory for $B_{2}$. This is because the vector representation of $C_{2}$ with dimension 4 corresponds to the spinor representation of $B_{2}$.

(iii) $B$ and D-series: This is the series of orthogonal groups $\operatorname{so}(n), B_{r}$ if $n=2 r+1$ and $D_{r}$ if $n=2 r$, for which there is an $N=1$ embedding of $s l(2)$ in the defining $n$-dim representation given by

$$
J_{0}=\frac{i}{2}\left(\begin{array}{ccc}
0 & 1 & \cdots \\
-1 & 0 & \cdots \\
\vdots & \vdots & \ddots
\end{array}\right), \quad J_{+}+J_{-}=i\left(\begin{array}{cccc}
0 & 0 & 0 & \cdots \\
0 & 0 & 1 & \cdots \\
0 & -1 & 0 & \cdots \\
\vdots & \vdots & \vdots & \ddots
\end{array}\right)
$$


that is purely imaginary and antisymmetric. As a consequence $\mathbf{g}_{0}=s o(n-2) \oplus u(1)$ and $\mathbf{h}=s o(n-3)$ commuting with $J_{+}+J_{-}$, which are represented respectively by

$$
i\left(\begin{array}{ccc}
0 & a & \cdots \\
-a & 0 & \cdots \\
\vdots & \vdots & b
\end{array}\right), \quad i\left(\begin{array}{cccc}
0 & 0 & 0 & \ldots \\
0 & 0 & 0 & \ldots \\
0 & 0 & 0 & \ldots \\
\vdots & \vdots & \vdots & c
\end{array}\right)
$$

where $a$ is a real number, $b$ is a real $(n-2)$-dim antisymmetric matrix and $c$ is a real $(n-3)$ dim antisymmetric matrix. The MIST in this case describe integrable perturbations of the WZW coset

$$
\frac{S O(n-2)}{S O(n-3)} \times U(1)
$$

and the decoupling condition (19) is always satisfied for any $n$. Hence, setting $\chi=$ 0 , which in the notation above corresponds to $a=0$, yields the WZW coset model $S O(n-2) / S O(n-3)$ plus an integrable perturbation that follows from the potential term $\operatorname{Tr}\left(g^{-1}\left(J_{+}+J_{-}\right) g\left(J_{+}+J_{-}\right)\right)$. Then, for all $n$, we obtain the associated so $(n)$ nonAbelian Toda theory by coupling to gravity according to the general framework.

The simplest non-trivial example is provided by $B_{2}=s o(5)$. After solving the WZW constraints (16), one finds that the massive integrable soliton theory for $\chi=0$ (see [12] for details) coincides with the complex sine-Gordon model for a complex field $u$ satisfying the equation of motion

$$
\bar{\partial} \partial u+\frac{u^{\star} \partial u \bar{\partial} u}{1-u u^{\star}}=m^{2} u\left(1-u u^{\star}\right) .
$$

This model has a residual $U(1)$ symmetry generated by $u \rightarrow e^{i \theta} u$ and provides a charged generalization of the ordinary sine-Gordon model. It has been identified with an integrable perturbation of the $S O(3) / S O(2)$ WZW model, where the potential term $|u|^{2}$ represents the first thermal parafermion operator in a Lagrangian framework [6, 7]. Setting $u=\sin r \exp (i \theta)$ we obtain a more standard description of the model in a curved background geometry with diagonal metric $\left(1, \tan ^{2} r\right)$. Upon gravitational dressing the resulting conformal theory is the $B_{2}$ non-Abelian Toda model that was also considered by Gervais and Saveliev in a different context [9]. We may introduce the non-compact version based on the WZW coset $S L(2) / U(1)$ by treating $u$ and $u^{\star}$ as the independent variables $u=\sinh r \exp (-\theta), u^{\star}=-\sinh r \exp (\theta)$. Then, the gravitationally dressed model takes the form (6). It is clear now that for higher values of $n$ we have multi-component generalizations associated to the perturbed WZW coset $S O(n-2) / S O(n-3)$ with the target space field $\mathbf{u}$ (and its conjugate $\mathbf{u}^{\star}$ ) having $n-4$ components. The non-Abelian Toda theory that arises after gravitational dressing in this case is summarized by the Lagrangian

$$
L^{\prime}=-\frac{1}{1-\mathbf{u} \mathbf{u}^{\star}}\left(\partial \mathbf{u} \bar{\partial} \mathbf{u}^{\star}+\bar{\partial} \mathbf{u} \partial \mathbf{u}^{\star}\right)+\partial \phi \bar{\partial} \phi+m^{2} e^{2 \phi}\left(1-2 \mathbf{u u}^{\star}\right)
$$

as it appears in [9]. 
Concluding this class of examples we mention that the $D$-series admits additional $N=1$ embedding when $r$ is an even number $2 p$ giving rise to MIST based on the WZW coset $U(2 p) / S p(p)$. This possibility, as well as the models based on exceptional algebras will not be considered here any further.

It is intriguing, as side comment, that the coset space geometry of the WZW models $S O(n-2) / S O(n-3)$ describe the dynamics of the physical degrees of freedom of a bosonic string propagating in $(n-1)$-dim flat Minkowski space after solving the classical Virasoro constraints in the $X^{0}=\tau$ gauge [14]. In this context the potential term $|\mathbf{u}|^{2}$ originates from self-interactions of the string à la Kalb-Ramond, as was originally pointed out in four dimensions by Lund and Regge [15]. The multi-component generalizations of the sine-Gordon model based on the WZW coset $S O(n-2) / S O(n-3)$ arise in yet another context by performing a conformal reduction à la Pohlmeyer of the ordinary 2dim non-linear $\sigma$-models based on $S^{n-2}=S O(n-1) / S O(n-2)$ [16]; for $n=4$ we obtain the sine-Gordon, for $n=5$ the complex sine-Gordon, and so on. It will be interesting to investigate the relevance of the gravitationally dressed models (41) to either of these two seemingly unrelated frameworks that also give rise to generalized sine-Gordon models.

One may inquire whether the classically conformal Toda theories, including their non-Abelian generalizations that result by gravitational dressing, define quantum mechanically consistent string backgrounds. The Toda potential can be regarded as an exactly marginal perturbation that has been added to the 2-dim $\sigma$-model, and as such it plays the role of the tachyon. If we consider the corresponding $\beta$-functions to lowest order in $\alpha^{\prime}$ we have to find a dilaton field $\Phi$ so that the conformal invariance is maintained. Since the presence of a tachyon potential $V$ does not affect $\beta\left(G_{\mu \nu}\right), \beta\left(B_{\mu \nu}\right)$ and $\beta(\Phi)$ to this lowest order, we may momentarily neglect $\beta(V)$ and examine first whether the non-Abelian Toda models have all other $\beta$ functions zero. In their formulation as gravitationally dressed multi-component sine-Gordon models (c.f. (41)) one finds that there is no consistent choice of dilaton that does the job (see for instance [17] and references therein). This might seem surprising bearing their original formulation (24) in terms of WZW models. The point is that there are two classically equivalent ways to formulate these theories, before or after solving the corresponding WZW constraints. Choosing the latter in order to connect directly with the generalized sine-Gordon models, like (40), it unavoidably leads to non-vanishing $\beta$-functions. In the other formulation the $\beta$ function equations are satisfied with a linear dilaton $\Phi=Q \phi$, but then the tachyon $\beta$-function equation $\beta(V)=0$ introduces a non-trivial algebraic relation between the numerical coefficient $Q$ and $\gamma$ that comes from the general conformal factor $e^{2 \gamma \phi}$; for example, this condition for the $B_{2}$ non-Abelian Toda theory reads [17]

$$
4 \gamma=Q \pm \sqrt{Q^{2}-\frac{4}{\alpha^{\prime}}}
$$

Thus, the quantization of the models in the unreduced formulation (24) is more appropriate for string applications, although further work is required to resolve some technical issues that are involved (analogous to Liouville theory for $c>1$ ). For a recent systematic exposition of Toda-like $\sigma$-model solutions of string theory see also [18]. 
A final technical point concerns the type of extended conformal symmetries that the models like (41) exhibit classically. Recall that the conformal coset with Lagrangian $\left(\partial \mathbf{u} \bar{\partial} \mathbf{u}^{\star}+\bar{\partial} \mathbf{u} \partial \mathbf{u}^{\star}\right) /\left(1-\mathbf{u} \mathbf{u}^{\star}\right)$ has a chiral $W_{\infty}$ symmetry generated by appropriate bilinear combinations of the parafermion currents. Turning on the potential $|\mathbf{u}|^{2}$ changes these local chiral conservation laws into non-chiral because the conformal invariance is broken but the integrability is preserved (see [6] for details on these models). The gravitational dressing restores classically the conformal invariance, but the curious thing is that the local conservation laws are not generated by $W_{\infty}$ anymore, rather by its consistent truncation restricted only to elements of even spin. For example, for the $B_{2}$ model there are three chiral currents of spin 2, one local that is provided by the stress-energy tensor and a pair of non-local fields, which are essentially the gravitationally dressed parafermions [9]. Hence, going a step further to construct all the local chiral conservation laws one has to consider appropriate bilinears of these parafermions, which yield $W$-generators with spin $2,4,6, \cdots$ in this case. This can be verified directly, but we spare the details of the computation. There might be a deeper relation of this result to the phase diagram of the sine-Gordon model (and probably its multi-component generalizations), where it was found that the overall velocity of the renormalization group flow is also cut in half by gravity $[4,5]$.

Summarizing, we have found that certain multi-component generalizations of the sine-Gordon model that arise as integrable perturbations of WZW cosets away from criticality turn into conformal non-Abelian Toda theories by coupling to 2-dim gravity. Although the backgrounds resulting by imposing classically the WZW constraints are not conformal quantum mechanically, their integrability should be preserved in the quantum theory. Our results offer another justification for focusing interest into the theory of non-Abelian Toda systems in future investigations, because of their relevance to 2-dim gravity; our viewpoint is different and perhaps complementary to other interpretations that were proposed before for such non-Abelian models [9]. An interesting question that arises in this context is the coupling of various multi-component sine-Gordon models to 2-dim gravity using Polyakov's chiral gauge as an alternative to the conformal gauge. Finally, it will be interesting to examine in detail the modifications introduced by 2-dim gravity on the renormalization group flow of multi-component sine-Gordon models, such as (40), and study possible gravitational phase transitions by exhibiting singularities of the corresponding partition functions. This will provide a systematic extension of previously known results on the gravitational sine-Gordon model $[4,5]$.

\section{Acknowledgements}

We thank H.J. Shin and M.V. Saveliev for useful discussions. Q.P. is supported in part by the program of Basic Science Research, Korean Ministry of Education BSRI-96-2442, and by the Korean Science and Engineering Foundation through CTP/SNU. He also wishes to thank the hospitality of CTP at MIT during the final stages of this work. 


\section{REFERENCES}

1. S. Coleman, "Dilations", in Aspects of Symmetry: Selected Erice Lectures, Cambridge University Press, 1985.

2. A. Zamolodchikov, "Integrable Field Theory from Conformal Field Theory", in Advanced Studies in Pure Mathematics $\underline{19}$ (1989) 1.

3. F. David, Mod. Phys. Lett. A3 (1988) 1651; J. Distler and H. Kawai, Nucl. Phys. B321 (1989) 509.

4. G. Moore, "Gravitational Phase Transitions and the Sine-Gordon Model", Yale preprint YCTP-P1-92, hep-th/9203061.

5. E. Hsu and D. Kutasov, Nucl. Phys. B396 (1993) 693; C. Schmidhuber, Nucl. Phys. B404 (1993) 342.

6. I. Bakas, Int. J. Mod. Phys. A9 (1994) 3443.

7. Q-H. Park, Phys. Lett. B328 (1994) 329; Q-H. Park and H.J. Shin, Phys. Lett. B347 (1995) 73; ibid B359 (1995) 125.

8. A. Leznov and M. Saveliev, Lett. Math. Phys. $\underline{6}$ (1982) 505; Comm. Math. Phys. $\underline{89}$ (1983) 59 .

9. J.-L. Gervais and M. Saveliev, Phys. Lett. B286 (1992) 271; A. Bilal, Nucl. Phys. B422 (1994) 258.

10. A. Bilal and I. Kogan, Nucl. Phys. B449 (1995) 569; A. Bilal, "Does Coupling to Gravity Preserve Integrability?", Ecole Normale preprint LPTENS-96/09, hepth/9601129.

11. E. Abdalla and M. Abdalla, Phys. Lett. B365 (1996) 41.

12. T. Hollowood, J. Miramontes and Q-H. Park, Nucl. Phys. B445 (1995) 451.

13. Q-H. Park and H.J. Shin, Nucl. Phys. B458 (1996) 327.

14. I. Bakas and K. Sfetsos, "Universal Aspects of String Propagation on Curved Backgrounds", to appear in Phys. Rev. D., hep-th/9604195.

15. F. Lund and T. Regge, Phys. Rev. D14 (1976) 1524.

16. I. Bakas, Q-H. Park and H.J. Shin, Phys. Lett. B372 (1996) 45.

17. A. Bilal, "Consistent String Backgrounds and Completely Integrable 2-D Field Theories", Ecole Normale preprint LPTENS-95/32, hep-th/9508062.

18. C. Klimcik and A. Tseytlin, Nucl. Phys. B424 (1994) 71. 